http://jmscr.igmpublication.org/home/ ISSN (e)-2347-176x ISSN (p) 2455-0450 crossref DOI: https://dx.doi.org/10.18535/jmscr/v7i11.03

\title{
Study of Clinical Outcomes of Cardiopulmonary Resuscitation in Children
}

\author{
Authors \\ Atul Tukaram Rane*, Shakuntala S Prabhu, Sumitra Venkatesh \\ Bai Jerbai Wadia Hospital for Children, Mumbai, India \\ *Corresponding Author \\ Atul Tukaram Rane \\ BJWHC, Acharya Donde Marg, Parel East, Mumbai 400012, India
}

\begin{abstract}
Introduction: It was a prospective observational cross sectional study conducted in tertiary care center.

Objective: To study the hemodynamics within 24 hours of initiation of cardiopulmonary resuscitation (CPR) and to compare the clinical \& resuscitative parameters with the outcome post CPR.

Method: Forty children aged 1 month tol5 years who required CPR in hospital were included in the study. Neurological status was classified as neurologically intact, impaired or dependent. The cohort was divided into long-term survivors, short-term survivors and non-survivors.

Results: Twenty four children required CPR for less than 15 minutes (60\%) and 23/40 (57.5\%) required resuscitation only once. Return of spontaneous circulation was seen in 35 patients (87.5\%). Shorter duration and few attempts at CPR gave better chances of survival. Oliguria prior to CPR led to significantly poor outcomes. Prolonged ventilation (>7days) was directly related to worse neurological impairment and dependency $(p=0.00054)$. Out of 40,7 patients $(17.5 \%)$ were non-survivors, $8(20 \%)$ were short-term survivors and $25(62.5 \%)$ were labeled as long-term survivors. Of 25 patients who survived till discharged 17 (68\%) were neurologically normal, 3 (12\%) had some functional neurological impairment.

Conclusions: Eighty two percent children survived post CPR with $68 \%$ having a normal neurological status on discharge. Shorter duration and fewer attempts of CPR, higher motor response post CPR and early institution of enteral feeds had a favorable impact on survival outcome. Oliguria, increased fluid requirement, hyponatremia, hyperglycemia, hypoxia and hypocarbia in the peri-resuscitation period were unfavorable factors for survival. Prolonged ventilator support correlated with poorer neurological outcome.

Keywords: Paediatric, Cardiopulmonary resuscitation, Outcomes, Survival.
\end{abstract}

\section{Introduction}

Primary cardiac arrest is rare in children and more commonly present as a secondary cardiopulmonary event which results in asphyxia and as a terminal event in other childhood illnesses like sepsis, shock etc. Many children have a relatively long 'prearrest' phase (i.e. cardiac arrest following prolonged physiological deterioration). Hence it is imperative to avert cardiopulmonary arrest by early recognition as the outcomes after cardiac arrest are dismal and those surviving are left with some neurological impairment.

Many patients who receive cardiopulmonary resuscitation (CPR) for cardiac arrest do not survive to leave hospital. Survivors of CPR have variable susceptibility to hypoxic-ischemic brain injury, depending on the duration of cardiopulmonary arrest, extent of resuscitation efforts, and underlying co morbidities. Better outcome after paediatric 
resuscitation is still a dilemma. When in-hospital respiratory arrest or failure is treated before the development of cardiac arrest, survival ranges from $60 \%$ to $97 \%$. Results of in-hospital resuscitation are better with an overall survival of $27 \%$. Paediatric patients were more likely to survive to discharge than adults $(6.4 \% \text { versus } 4.5 \%)^{[1]}$. However the contributing factors to such a dismal outcome are still very unclear.

This study planned to collect, access and analyse demographic variables, vital parameters and laboratory findings in correlation to these outcomes in children requiring CPR. It would also create a scope for analysing any possible methods which could help improve survival of such children.

\section{Methods}

This study was a prospective observational cross sectional study conducted in a tertiary referral care centre exclusively for children.

All children aged 1 month to 15 years admitted inhouse (Intensive paediatric care unit (IPCU), inpatient and emergency department) requiring CPR during January 2013 to June 2014 were screened to be included in the study. 40 cases fulfilled the inclusion criteria and were prospectively followed up. Birth asphyxiated neonates, children with underlying heart disease, children with "Do not resuscitate order" (DNR) given, children requiring CPR out of hospital prior to admission and children who were discharged against medical advice (DAMA) prior to completion of therapy were excluded from study.

The study was approved by the institutional ethics committee and informed consent was obtained from the parent/guardian of all patients who were included in study.

The course in the hospital and all resuscitation details were recorded.

Patient's demographic variables were recorded including age and gender. Their chief complaints were categorized as per the predominant system affection (respiratory/renal/gastrointestinal/ neurological) and whether single system was affected or multiorgan involvement present at the time of admission. Illness category and any specific event causing or precipitating the cardiopulmonary arrest were taken note of. Illness category was classified on the basis of respiratory, cardiac, nervous, renal, haematological and others. Event predisposing to requirement of CPR was classified as respiratory failure / cardiac / cardiopulmonary.

All patients requiring resuscitation were resuscitated as per PALS Guidelines of 2010 given by American Health Association and all standard protocols followed. $^{[2]}$

During analysis of CPR details attention was given to the level of expertise of the person initiating the resuscitation (IPCU Registrar/Ward Registrar/ Casualty officer), place of resuscitation (IPCU/ Casualty/Ward, duration of $\mathrm{CPR}$ required $(<15$ $\min / 15-30 \mathrm{~min} />30 \mathrm{~min}$ ), whether child was transferred to the IPCU (Yes/No), Time required for IPCU transfer of the child was noted (If at all transferred $<30 \mathrm{~min}$ or $>30 \mathrm{~min}$ ). Number of CPR attempts required $(1 /=>2)$. If fluid boluses were required during resuscitation, it was noted and recorded for comparison.

Examination findings like Sensorium (good/poor), Temperature (febrile/afebrile), Heart rate (tachycardia/bradycardia/normal), Respiratory rate (tachypnoea/bradypnea/normal), Peripheral pulses (Well felt/feeble), Capillary refill time (CRT) (normal/delayed), Pulse oximetry prior to CPR (<90\% / 90-93\% / >94\%), Blood pressure (Normal/ High/Low) of the resuscitated patients were noted. Whether invasive BP monitoring and central venous pressure (CVP) monitoring was required and used was also noted. Mean arterial pressure, Urine output (oliguria/normal), Presence of any occult or manifested bleeding, Presence of any gastric aspirates in the post CPR hospital stay, Motor response as per Glasgow coma scale was noted.

Return of spontaneous circulation (ROSC) immediately post CPR was noted.

All investigations were checked and recorded and some relevant parameters were selected for analysis. Haemoglobin values (gm/dl) $(<7 / 7-10 />10)$, Haematocrit values $(\%)(<25 / 25-30 / 30-35 />35)$, Platelet counts (lakhs/cu.mm) $(<1.5 / 1.5-4.5 />4.5)$, 
Liver transaminases (SGPT and SGOT) $(<40 / 40-$ 200 />200), Renal parameters (BUN/ Serum Creatinine) (high or normal).

Blood gas analysis was done. Parameters like $\mathrm{pH}$ $(<7.35 / 7.35-7.45 />7.45)$, PCO2 (mmHg) $(<40 /$ $>40), \mathrm{PO} 2(\mathrm{mmHg})(<100 / 100-200 />200), \mathrm{HCO} 3$ $(\mathrm{mmol} / \mathrm{L})(<16 / 16-24 />24)$, SPO2 (\%) $(<94 />94)$ were noted.

Other values of Serum Calcium levels $(\mathrm{mg} / \mathrm{dl})(<9 /$ $>9)$, Random blood sugar (RBS) (<45/45128/>128), Sodium (Na) levels (mmol/L) (<135 / 135-145/>145), Potassium (K) levels $(\mathrm{mmol} / \mathrm{L})$ (<3.5/ 3.5-5.5 />5.5). Serum lactate levels $(\mathrm{mmol} / \mathrm{L})$ (0.8-1.8) were used for metabolic screening.

ECG recordings on the monitor were recorded just antecedent to CPR and noted. (Asystole/ Bradycardia/Ventricular tachycardia/within normal limits)

X-Ray findings were noted if suggestive of pneumonia, whether findings were bilateral and whether there was any cardiomegaly on the X-Ray (cardiothoracic ratio $>0.55$ ).

The mode (pressure/volume) and duration of ventilation was noted. Duration of ventilation was classified as ( $<1$ day / 1-7 days / > 7 days). Whether the patient was given ulcer prophylaxis and /or enteral feeds post CPR was documented and used for comparison with the outcomes.

The outcome of the resuscitation was recorded and the post resuscitation survival duration till discharge from the hospital was documented. Their final diagnoses entered. Analysis was further done on organ dysfunction. On discharge neurological functional status was studied and classified as neurologically intact, impaired or dependent. The cohort was divided into three groups: long-term survivors (survived to discharge); short-term survivors (survived longer than 24 hours after CPR but not until discharge) and non-survivors (died within 24 hours of their arrest).

These findings were documented on the case Performa and each patient's parameters were tabulated for analysis. Each patient was followed up till discharge.

\section{Statistical Analysis}

Qualitative data was represented in form of frequency and percentage.

Association between qualitative variables was assessed by Chi-Square test with Continuity Correction for all 2X2 tables and Fisher's exact test for all $2 \mathrm{X} 2$ tables where $\mathrm{p}$-value of Chi-Square test was not valid due to small counts. Adjacent row data of more than 2X2 tables was pooled and ChiSquare test reapplied in case more than $20.0 \%$ cells had expected count less than 5 .

Quantitative data was represented using mean \pm SD and Median \& IQR (Interquartile range).

Analysis of Quantitative data between Non-survivor $\&$ Survivor cases was done using unpaired t-test if data passed 'Normality test' and by Mann-Whitney Test if data failed 'Normality test'.

Binary Logistic Regression was performed between Outcome (Non-survivor) as dependent variable and a set of independent (predictor) variables.

SPSS Version 17 was used for most statistical analysis and Microsoft Excel 2010 for graphical representation.

\section{Results}

Out of 40 cases $22(55 \%)$ were less than 1 year of age. The mean age in non-survivors was 3.17 years $(\mathrm{SD}=3.81$, median=0.6) and the mean age in survivors was 2.74 years $(\mathrm{SD}=3.5$, median=1). There was an incidental male preponderance noted with ratio of 5:3.

Sixty percent patients had single system involvement on presentation, while $40 \%$ cases came with multiorgan manifestations. Respiratory complaints were most common (65\%). Respiratory system was the predominant illness category in 14 cases $(35 \%)$. Respiratory arrest was the more common event requiring CPR in $34(85 \%)$, with 3 (7.5\%) having primary cardiac arrest and $3(7.5 \%)$ with both cardiopulmonary compromise.

Twenty four children required CPR for less than 15 minutes (60\%), $10(25 \%)$ for $15-30$ minutes and 6 children (15\%) for more than 30 minutes. The mean duration in survivors was $13.20 \mathrm{~min}(\mathrm{SD}=12.49$, median=10 $\mathrm{min})$. And the mean in non-survivors 
was $36.67 \mathrm{~min}$ (SD 40.65, median=15 $\mathrm{min}$ ). Twenty three of forty $(57.5 \%)$ required resuscitation only once while the remainder $17(42.5 \%)$ required 2 or more CPR efforts during their hospital stay.

Nineteen patients $(47.5 \%)$ required fluid boluses during resuscitation.

Thirty three $(82.5 \%)$ subjects had poor sensorium on presentation or just prior to CPR, 15 (37.5\%) were febrile, $42.5 \%$ had tachycardia and $15 \%$ had bradycardia, $27(67.5 \%)$ had abnormal breathing rates. It was seen that $23(57.5 \%)$ cases had good volume pulses, 24 (60\%) had normal CRT. By pulse oximetry 20 cases $(50 \%)$ had saturation $<94 \%$ prior to $\mathrm{CPR}$. The mean value was $90.48 \%$ ( $\mathrm{SD}=8.30$, median=90) in survivors, and $90.67 \%(\mathrm{SD}=12.01$, median $=94)$ in non survivors. Six $(15 \%)$ patients had hypotension prior to CPR. Seven of twenty three $(30.4 \%)$ subjects were monitored with invasive methods. Twelve of twenty three (52.2\%) patients were monitored with central venous pressure (CVP) lines in IPCU. Ten cases (25\%) had oliguria prior to $\mathrm{CPR}$ and required volume resuscitation. Six (15\%) had bleeding manifestations, $4(10 \%)$ patients had gastric aspirates, 9 patients $(22.5 \%)$ had seizures. Seven patients (17.5\%) had response less than M4 and $33(82.5 \%)$ had motor response of M4 or more post CPR. Return of spontaneous circulation was seen in 35 patients $(87.5 \%)$ post CPR.

Mean $\mathrm{Hb}$ in survivors was $9.38 \mathrm{~g} / \mathrm{dl}(\mathrm{SD}=1.92)$ and mean in non-survivors was $9.64 \mathrm{~g} / \mathrm{dl}(\mathrm{SD}=2.43)$. The mean haematocrit in survivors was $29.84 \%$ $(\mathrm{SD}=5.89)$ and in non survivors was $29.87 \%$ ( $\mathrm{SD}=6.85)$. Eight $(20.5 \%)$ had platelets less than 1.5 $\mathrm{lakh} / \mathrm{mm}^{3}$. Liver enzyme derangement was seen in approximately $60 \%$ cases, renal parameter impairment in $29.6 \%$ cases, blood $\mathrm{pH}$ abnormalities in $59 \%$ cases.

The mean $\mathrm{PO}_{2}$ in survivors was $133.7 \mathrm{mmHg}$ $(\mathrm{SD}=54.98)$ and in non survivors was $23.85 \mathrm{mmHg}$ $(\mathrm{SD}=73.57)$. Fifteen $(38.5 \%)$ patients had $\mathrm{HCO}^{3}$ less than $16 \mathrm{mmol} / \mathrm{L}$.

Thirty $(76.9 \%)$ subjects had $\mathrm{PCO}_{2}$ levels less than $40 \mathrm{~mm} \mathrm{Hg}$. The mean in survivors was $5.63 \mathrm{mmHg}$
$(\mathrm{SD}=15.16)$ (median=32.10) and in non survivors was $26.86 \mathrm{mmHg}(\mathrm{SD}=9.71$, median=23.75).

On ABG, 31 (79.5\%) subjects had saturation of more than $94 \%$ and $8(20.5 \%)$ had values of less than $94 \%$. The mean value in survivors was $96.57 \%$ $(\mathrm{SD}=5.08)$ (median=99.0\%) and in non survivors was $94.59 \%$ ( $\mathrm{SD}=4.91)$ (median=97\%).

The mean serum calcium value in survivors was $9.16 \mathrm{mg} / \mathrm{dl}(\mathrm{SD}=0.92)$ and in non survivors was $8.87 \mathrm{mg} / \mathrm{dl} \quad(\mathrm{SD}=0.80) .3$ children $(7.7 \%)$ had hyperglycaemia. Mean RBS in survivors was $96.4 \mathrm{mg} / \mathrm{dl}(\mathrm{SD}=18.06$, median=90) and the mean in non survivors was $99.07 \mathrm{mg} / \mathrm{dl} \quad(\mathrm{SD}=71.2$, median=83). $12 \quad(30.8 \%)$ had hyponatremia $(\mathrm{Na}<135)$. Mean value in survivors was 137.52 $\mathrm{mmol} / \mathrm{l}(\mathrm{SD}=5.72, \quad$ median=138) and in non survivors was $132.07 \mathrm{mmol} / \mathrm{l} \quad(\mathrm{SD}=8.56$, median=134.5). The mean potassium $(\mathrm{K})$ value in survivors was $4.43 \mathrm{mmol} / \mathrm{l}(\mathrm{SD}=0.83)$ and in non survivors was $4.47 \mathrm{mmol} / \mathrm{l}(\mathrm{SD}=0.86)$. Eight of fifteen $(53.3 \%)$ had elevated lactate levels. 9/33 $(27.3 \%)$ had abnormal ECG recordings in the form of asystole, ventricular tachycardia and sinus bradycardia. xii) X-Ray chest showed pneumonia in $18(45 \%)$ subjects, bilateral haziness in $12(30 \%)$ cases, and cardiomegaly in $3(7.5 \%)$ cases with some overlapping findings.

Eleven $(27.5 \%)$ were intubated and ventilated for duration of less than 24 hours. Sixteen (40\%) were ventilated for 2-7 days and $13(32.5 \%)$ required ventilator support for more than 7 days (prolonged ventilation).

Twenty one $(52.5 \%)$ required inotropic support. Eighteen $(45 \%)$ required packed cell transfusions, $28(70 \%)$ patients were given ulcer prophylaxis. Enteral feeds were given to $32(80 \%)$ subjects who received CPR.

Non survivors were 7 (17.5\%). The mean value in survivors was 32.6 days $(\mathrm{SD}=35.68$, median=23) and in non survivors was 11.49 days $(\mathrm{SD}=23.16$, median=3). Eight $(20 \%)$ were short-term survivors (survived longer than 24 hours after CPR but not until discharge) and $25(62.5 \%)$ were labelled as long-term survivors (survived to discharge). Of 25 patients who survived till discharged 17 (68\%) were 
neurologically normal, 5 (20\%) were dependent for their day to day activities on other family members and $3(12 \%)$ had some functional neurological impairment in form tone abnormalities or nerve palsies or limb paresis.

Thirty three of forty (82.5\%) children survived post CPR with $68 \%$ having a normal neurological status on discharge. The mean duration of hospitalization was 33 days. On univariate analysis; shorter duration of resuscitation, fewer attempts of CPR, early return of spontaneous circulation, higher motor response and early institution of enteral feeds post CPR had a favourable impact on survival outcomes $(\mathrm{p}<0.005)$.

Oliguria, increased fluid requirement, hyponatremia, hyperglycaemia, hypoxia and hypocarbia in the peri resuscitation period were unfavourable factors for survival $(p<0.005)$. Prolonged ventilator support also correlated with poorer neurological outcome at discharge.

Binary logistic regression analysis between outcome (Non survivor) as a dependent variable and a set of independent (predictor) variables was done.

The predictor variables used were age, presence of infection, presence of multi-organ involvement, respiratory event causing secondary cardiac arrest and number of CPR attempts. Out of all number of CPR was found to be the only statistically significant predictor of being a non-survivor at discharge. So it can be concluded that more the number of CPR attempts more the chances of being a non-survivor post CPR.

\section{Discussion}

We analysed the impact of different patient variables on survival outcomes and also influence of various resuscitative measures and post resuscitation care on neurological outcome at discharge and patient survival post CPR. 33/40 $(82.5 \%)$ children survived post CPR with $68 \%$ having a normal neurological status on discharge and $3(12 \%)$ had some form of functional neurological impairment. Five cases $(20 \%)$ were dependent for their day to day activities on other family members.
Eighty five children requiring CPR had respiratory arrest and not cardiac arrest. However the outcome post CPR did not show any significant association with the event precipitating the cardiopulmonary arrest. $\quad(p=0.654)$. A study ${ }^{[3]}$ previously done showed that cardiac arrest often develops as a progression of respiratory failure and when this is treated before the development of cardiac arrest, survival ranges from $60-97 \%$.

Somehow the expertise of person giving CPR or the place of CPR did not affect the outcome of survival $(p=0.311)$. This was probably because of universal training of all medical and paramedical staff in basic life support skills; hence the quality of CPR at all times would have been optimum. A study had concluded that survival post CPR could improve with high rate and degree of training at the time of their employment. ${ }^{[4]}$

As per our study no particular history or examination finding had an impact on survival outcome of CPR. However duration of CPR and number of attempts required during total hospital stay did matter. This correlates to the shorter duration of hypoxia and ischemia to the vital organs i.e. the brain and heart and hence affects chances of positive outcome.

Various lab parameters like hyponatremia, hyperglycaemia, and low $\mathrm{SPO}_{2}$ had an impact as mentioned before. Analysing the records it was found that there was significant association between the child's urine output and the outcome. Those with oliguria had poor outcomes compared to survivors who had adequate urine output prior to CPR ( $\mathrm{p}=0.024)$.

So it is concluded that decreased urine output which is an indicator of hypovolemia and poor renal perfusion was a poor prognostic indicator for survival outcome of children in this study.

Previous studies had not found any direct correlation to the urine output with survival outcome in contrast to this study.

Seven patients $(17.5 \%)$ had response less than M4 and the $33(82.5 \%)$ had motor response of M4 or more post CPR. On analysis this study showed that higher the motor response better was the survival 
outcome post CPR ( $\mathrm{p}=0.00017)$. Those cases with response less than $\mathrm{M} 3$ did not survive to discharge compared to those with motor response more than or equal to M4. Hence the motor response can be used as an easy to do test for prognosticating the survival post CPR. [Table 2.A and 2.B]

The American Academy of Neurology also had studied that the prognosis is invariably poor in comatose patients with absent pupillary or corneal reflexes, or absent or extensor motor responses 3 days after cardiac arrest. ${ }^{[5]}$

A study done in Taiwan in $2009^{[6]}$ showed $60.7 \%$ achieved sustained return of spontaneous circulation, $19.8 \%$ survived to discharge and $15.5 \%$ had favourable neurological outcome. In our study out of 40 cases, $35(87.5 \%)$ had return of spontaneous circulation, $25(62.5 \%)$ children survived to discharge out of which 17 were neurologically normal.

The duration of ventilation did not affect the survival on discharge post CPR $(p=0.681)$ but when it was studied in comparison with functional status on discharge in the long term survivors; it was found that more the ventilation duration; more is the neurological impairment and dependency.

It was found after analysis that those patients given enteral feeds had higher chances of survival till discharge as compared to those not started on enteral feeds.

There was statistically significant association present between this variable and survival outcome at discharge. Another previous study does mention that in patients surviving $24 \mathrm{hrs}$ after CPR, initiation of enteral nutrition within $48 \mathrm{~h}$ and maintenance of normoxia were associated with a positive outcome. [7]

Our study shows some few new findings not reported commonly before like impact of oliguria on poor survival post CPR. Motor score of more than or equal to 4 (GCS), can be used as a surrogate marker for survival post CPR. Also early introduction of enteral feeding may have a positive influence on survival outcome.

Limitations: small sample size. Long term neurological assessment needs to be done post discharge for better understanding of nervous system prognostication.

Table 1 Outcome at discharge

\begin{tabular}{|c|c|c|}
\hline Outcome & Number & Percentage \\
\hline \multicolumn{3}{|l|}{ Survivability } \\
\hline Non-survivors ${ }^{*}$ & 7 & $17.5 \%$ \\
\hline Short-term survivors ${ }^{\#}$ & 8 & $20.0 \%$ \\
\hline Long-term survivors $\$$ & 25 & $62.5 \%$ \\
\hline \multicolumn{3}{|c|}{ Neurological Function Status of Long Term Survivors } \\
\hline Normal & 17 & $68.0 \%$ \\
\hline Impairment & 3 & $12.0 \%$ \\
\hline Dependant & 5 & $20.0 \%$ \\
\hline
\end{tabular}

*: died within 24 hours of cardiac arrest; \#: survived longer than 24 hours after CPR but not until discharge; $\$$ : survived to discharge.

Table 2.A Motor response and outcome

\begin{tabular}{|c|c|c|}
\hline \multirow{2}{*}{$\begin{array}{l}\text { Motor } \\
\text { Response }\end{array}$} & \multicolumn{2}{|c|}{ Outcome } \\
\hline & Non-survivor (15) & Survivor (25) \\
\hline M1 (3) & 3 & 0 \\
\hline M2 (1) & 1 & 0 \\
\hline M3 (3) & 3 & 0 \\
\hline M4 (5) & 3 & 2 \\
\hline M5 (15) & 5 & 10 \\
\hline M6 (13) & 0 & 13 \\
\hline
\end{tabular}

Table 2.B Association among the cases betweenmotor response and outcome

\begin{tabular}{|c|c|c|}
\hline Chi-Square Tests & Value (df) & p-value \\
\hline Pearson Chi-Square \$ & $20.658(5)$ & 0.00094 \\
\hline Pearson Chi-Square ^ ${ }^{\wedge} \#$ & $12.698(1)$ & 0.00037 \\
\hline Fisher's Exact Test ^ , \# & & 0.00017 \\
\hline \multicolumn{3}{|c|}{$\begin{array}{l}\$ 9 \text { cells }(75.0 \%) \text { have expected count less than } 5 . \wedge \text {, \# Row } \\
\text { data pooled \& Chi-Square Test reapplied with Continuity } \\
\text { Correction. }\end{array}$} \\
\hline \multicolumn{3}{|c|}{$\begin{array}{l}\wedge \text {, \# } 1 \text { cell }(25.0 \%) \text { has expected count less than } 5 . \text { P-value } \\
\text { of Fisher's test will be used. }\end{array}$} \\
\hline
\end{tabular}

\section{Sources of support in the form of grants: Nil}

\section{References}

1. J Herlitz et al: Characteristics of cardiac arrest and resuscitation by age group: an analysis from the Swedish Cardiac Arrest Registry. Am J Emerg Med. 2007;25:10251031

2. Recognition and management of cardiac arrest. PALS provider's manual (Professional) 2010

3. Sahu $\mathrm{S}$ et al: Better outcome after pediatric resuscitation is still a dilemma: J Emerg 
Trauma Shock. 2010 Jul-Sep; 3(3): 243250.

4. M Chakravarthy, S Mitra, L Nonis, N Yellappa. Outcome of in-hospital, out of intensive care and operation room cardiac arrests in a tertiary referral hospital in India: Comparison of outcomes of two audits. Indian J Anaesth 2014;58:479-81

5. E F Wijdicks et al. Practice parameter: prediction of outcome in comatose survivors after cardiopulmonary resuscitation (an evidence-based review): report of the Quality Standards Subcommittee of the American Academy of Neurology. Neurology. 2006;67:203-210.

6. Wu-En Fing, Li Meng-Ju, Huangshu-chien, Wang ching-chia Liu, Yuch-Ping, Lufrank Leigh et al; Survey of outcome of CPR in pediatric in-hospital cardiac arrest in a medical centre in Taiwan. Resuscitation 2009; 80(4) ; 443-448.

7. H K Lee, H Lee, J M No, Y T Jeon, J W Hwang, Y J Lim, H P Park. Factors influencing outcome in patients with cardiac arrest in the ICU. Acta Anaesthisol Scand.2013 Jul;57(6):784-92. 